\title{
Neural networks applicated in computer network security evaluation and reliability analysis
}

\author{
Zhao jinping \\ Nanchang University College of Science and Technology, Nanchang, Jiangxi Province, Beijing East \\ Road, No. 339, 330029 \\ 346591653@163.com
}

Keywords: BP neural network; computer network; network security evaluation; systematic review

\begin{abstract}
Computer network in the information age is undoubtedly an important bearing basis, it has become an important guarantee for the healthy functioning of all walks of life, in this context of computer network security has been the concern of the community. Computer networks in the development process its effective safety evaluation has become an indispensable work, and neural network is an important and effective evaluation tool in the process of evolving neural network, which is also the application surface getting bigger. Article on neural network in computer network security evaluation were analyzed and put forward relevant views. This paper establishes the index system of evaluation of computer network security, Computer network security evaluation method based on BP neural network is designed to build the evaluation model by MATLAB simulation results show that the method is feasible.
\end{abstract}

\section{Introduction}

With the rapid development of computer and network technology and extensive application of factors affecting more and more network security, network security issues become more complex, which requires the use of the system and network managers must be able to secure the network [1-3]. The degree of effective measurement, network security have a clear understanding of the exact extent of network security control systems and the factors affecting safety in order to protect the safety of the system to the maximum extent. Important ways and means of network security evaluation is to solve this problem $[4,5]$.

From a macro point of view the current computer has completely permeated society which, in all walks of life have an important role as a computer network environment running on the basis of environmental, safety issues and more and more attention [6]. In fact many network security constraints, including not only the invading virus, but also affected its own vulnerabilities, and these constraints there is a close link between interrelated strong, reflecting the complex, non-changeable linear relationship, which makes network security there have been many uncertainties, and to the smooth implementation of the relevant evaluation has brought a degree of retardation. The neural network is currently the most widely used one of a class of network security evaluation tools, BP neural network gradient descent algorithm as the basis, the characteristic is more significant, so the wide range of applications [7-9].

Compared with traditional network neural network embodies the following characteristics: linear. Under normal circumstances the neural network needs to provide a periodic training process, after the completion of the training process can be more ideal efficiency. Fault tolerance. Compared with the traditional model in terms of the neural network is highly fault-tolerant, and does not show irritation even when insufficient information integrity situation. The reason is mainly because of the presence of the above characteristics of neural network nodes are generally the local problem or feature among the nodes are identified and reflected the situation input node defects will not have integrated features of neural networks have an impact.. Neural network environment has a good affinity with the match, reflecting a strong ability to adapt. In some cases the neural network is in the input mode or output 
mode state, and by self-training neural networks have a "memory" role, and thus lends itself to maintain stable operating state.

\section{The basic principle of neural network}

The basic structure of the nervous system neurons (nerve cells), it is the basic unit of processing of mutual transmission of information between the various parts of the body [10]. According to the research results show that neural biologist, 1000 to 1011 neurons generally have the human brain. Each neuron consists of a cell body, a connecting to other neuronal axons projecting outward and some other shorter branches -- dendrites. The axon is the function of the output signal of the neurons is transferred to other neurons. Many nerve endings at the end of that excitement can simultaneously to multiple neurons. The function is received from other neurons. All the signals in the cell body of a neuron will receive the simply treated by axonal output. As shown in Figure 1 is the basis for most neural network model.

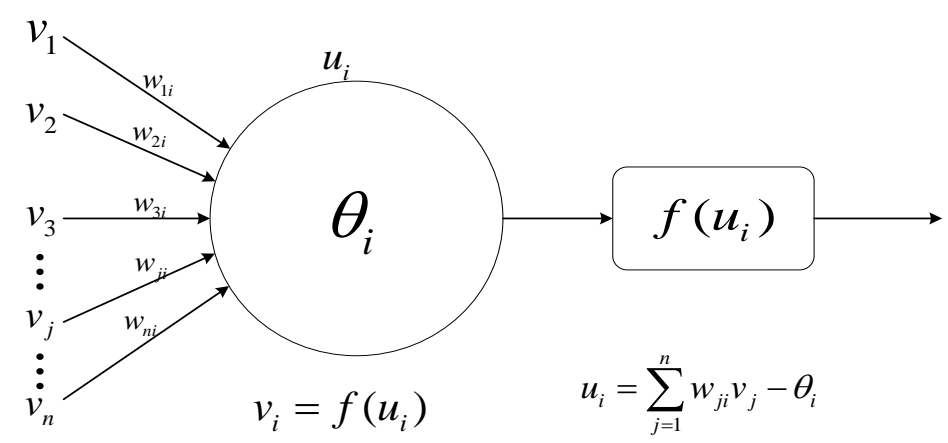

Figure 1. The basis for most neural network model.

The neurons themselves threshold for export, the output can be expressed as:

$$
Y=f\left(u_{i}\right)=f\left(\sum_{j=1}^{n} \omega_{j i} v_{j}-\theta_{i}\right)
$$

ui representative neuron i active value, namely the neuron state; function expression of the characteristics of the input and output neurons. In the M-P model, factory is defined as a step function:

$$
\begin{gathered}
v_{i}=f\left(u_{i}\right)= \begin{cases}1, & u_{i}>0 \\
0, & u_{i} \leq 0\end{cases} \\
z_{p k}=f\left(\text { net }_{p k}\right)=f\left(\sum_{i=0}^{n} v_{k i} x_{p i}\right), \\
y_{p j}=f\left(\text { net }_{p j}\right)=f\left(\sum_{k=0}^{q} \omega_{j k} z_{p k}\right), \quad k=0,1, \cdots, q
\end{gathered}
$$

\section{Networking security evaluation index system}

\section{Indicators for evaluating set}

Computer network is a complex system, many factors that affect network security, the possibility of establishing a scientific and rational network security evaluation, related to the ability to play a role and function of evaluation. Based on deep understanding summed safety factors, physical security and logical security level for the index evaluation system. Layer thinning of a complete network security evaluation system, was shown in Table 1. 
Table 1 Computer Network Security Evaluation System

\begin{tabular}{ccc}
\hline Aims & Level indicators & Secondary indicators \\
\hline & Security management & System security organization A1 \\
& Safety management System A2 \\
& Security personnel training A3 \\
& Physical security B & Anti-electromagnetic leakage measures \\
& & B1 \\
Computer & & Network room security B2 \\
Network & & Security of electricity supply B3 \\
Security & Line safety B4 \\
Assessmen & Fault-tolerant redundancy B5 \\
t & Safety equipment B6 \\
& data backup C1 \\
& Data recovery C2 \\
& Systems audit C3 \\
& Access control C4 \\
& Software security C5 \\
& digital signature C6 \\
& Anti-virus measures C7 \\
& Data encryption C8 \\
& Intrusion prevention C9 \\
\hline
\end{tabular}

\section{Design of computer network security evaluation model based on BP neural network}

As a general rule BP neural network design, the number of indicators and evaluation of the number of neurons in the input layer nodes, respectively. Evaluation system designed in this paper a total of 19 secondary indicators, and this article is based on secondary indicators for data collection, so the evaluation of the input layer model of a total of 19 input neurons nodes, each with two indicators one by one. According to prove Robert Hecht Nielson, for any closed interval in a continuous function can use a hidden layer of BP network to approximate, a three-layer BP neural network can complete any $\mathrm{N}$-dimensional mapping of the $\mathrm{M}$-dimensional, so in practice, most of the BP network with single hidden layer in the designed network also adopted this single hidden layer structure. In the BP neural network design, the network hidden layer nodes were few options, non-linear mapping function and fault-tolerant network is poor, too many nodes choose, so that will increase learning time, learning error is not necessarily the best, affect the learning efficiency, more empirical formula currently on the hidden layer nodes identified.

Since the BP neural network initial weights of the layers is arbitrary, it must first be trained neural network, so that the computer network security evaluation results deviation of the actual output value and the desired value as small as possible. Different scale computer network security evaluation this paper in advance collected 30 bodies of relevant data in Table 3 in which the partial data and the expected results. After the standardization process, in which 27 sets of data samples used to train as a learning network model designed in this paper, after 777 steps to reach the network training error tolerance requirements set, as shown in Figure 2. 


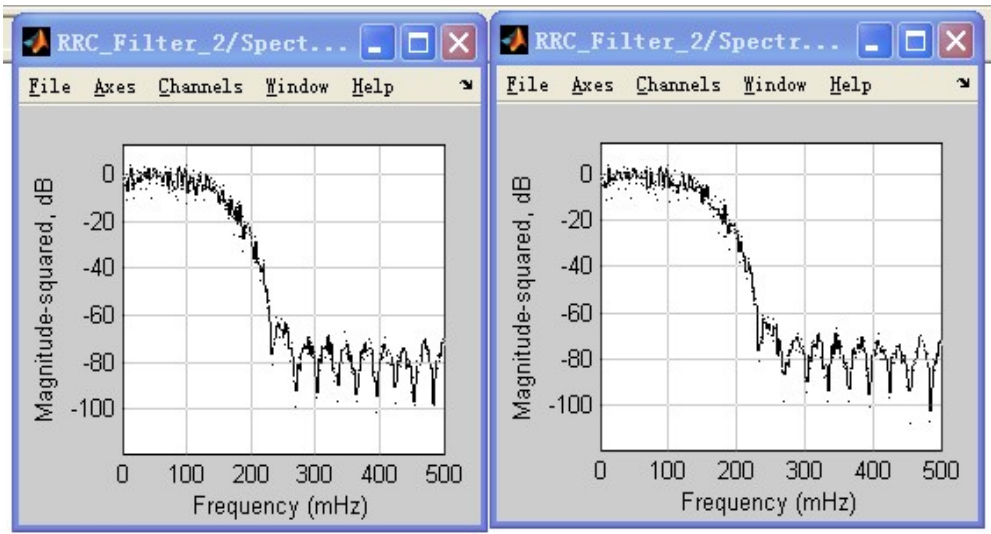

Figure 2. The network training error tolerance requirements set

\section{Summary}

Introduction of BP neural network model to evaluate the security of computer networks can overcome the disadvantages of the conventional evaluation method, the evaluation process to get rid of the uncertainty and randomness of subjective evaluation of personnel and in the understanding of ambiguity, to ensure that the results of the evaluation objective and accurate, thus providing an effective method for the evaluation of computer network security management. Neural network computer network security evaluations provide great technical support, through the application of neural network system to build a reliable evaluation system, in order to ensure the stable operation of the network in the state, prompting the network services can be implemented smoothly.

\section{Acknowledgement}

The authors would like to thank the Jiangxi reform project, NO: JXJG-14-28-2.

\section{References}

[1] Baoming Wu, Yonghui Guo, Bingxi Wang, "English Chinese machine translation rule based sentence structure analysis and transformation", Journal Information Engineering University, vol. 8, no. 1, pp. 30-33, 2007.

[2] Xinyu Dai, Cunyan Yin, Jiajun Chen, Guoliang Zheng, " Current situation and prospect of research on machine translation ", Computer Science, vol. 31, no. 11, pp. 176-184, 2004.

[3] Yao Meng, Tiejun Zhao, Sheng Li, Jianming Yao, " Based on the evaluation of the English syntactic structure disambiguation and self evaluation rule correction", Journal of Computer Research and Development, vol. 39, no. 7, pp. 802-808, 2002.

[4] He Yan Huang, Zhao Xiong Chen, "Based on analysis of complex long sentence translation algorithm ", Journal of Chinese information processing, vol. 16, no. 3, pp. 1-2, 2002.

[5] Li Shutao, Wang Yaonan, Zhang Changfan, "Objective evaluation and analysis of multisensor image fusion", Chinese Journal of Scientific Instrument, vol. 23, no. 6, pp. 651-654, 2002.

[6] WangJue, WangRen, MiaoDuoqian, "Data enriching based on rough set theory", Journal of Environmental Sciences, vol. 29, no. 3, pp. 63-69, 2010.

[7] Zheng Zhonglong, Zhao Jianmin, Yang Jie. "NMF with Log Gabor Wavelets for visualization". Lecture Notesin Computer Scienee, vol. 3691, no. 2, pp. 26-32, 2005.

[8] WangJue, WangRen, MiaoDuoqian, "Data enriching based on rough set theory", Journal of Environmental Sciences, vol. 29, no. 3, pp. 63-69, 2010.

[9] Q.R. Li, L.Y. Wei, S.F. Ma, “The Model Analysis of Vehicles Situation and Distribution in Intersections Abased on Markov Process," IEEE International Conference on Intelligent Transportation Systems. vol. 2, no. 10, pp.1076-1080, 2003.

[10]Barash D, Comaniciu A. "A common framework for nonlinear diffusion, adaptive smoothing bilateral filtering and mean shift ", Image Vision Computing, pp. 73-81, 2004. 